\title{
Non-rigid Multimodal Image Registration Based on the Expectation-Maximization Algorithm
}

\author{
Edgar Arce-Santana ${ }^{\star}$ Daniel U. Campos-Delgado, Flavio Vigueras-Gómez, \\ Isnardo Reducindo ${ }^{\star \star}$, and Aldo R. Mejía-Rodríguez ${ }^{\star \star \star}$ \\ Facultad de Ciencias, Universidad Autónoma de San Luis Potosí, \\ Av. Salvador Nava Mtz. S/N, Zona Universitaria, 78290, \\ San Luis Potosí, SLP, México \\ arce@uaslp.mx
}

\begin{abstract}
In this paper, we present a novel methodology for multimodal non-rigid image registration. The proposed approach is formulated by using the Expectation-Maximization (EM) technique in order to estimate a displacement vector field that aligns the images to register. In this approach, the image alignment relies on hidden stochastic random variables which allow to compare the intensity values between images of different modality. The methodology is basically composed of two steps: first, we provide an initial estimation of the the global deformation vector field by using a rigid registration technique based on particle filtering, obtaining, at the same time, an initial estimation of the joint conditional intensity distribution of the registered images; second, we approximate the remaining deformations by applying an iterative EM-technique approach, where at each step, a new estimation of the joint conditional intensity distribution and the displacement vector field are computed. The proposed algorithm was tested with different kinds of medical images; preliminary results show that the methodology is a good alternative for non-rigid multimodal registration.
\end{abstract}

\section{Introduction}

Image registration is an important task in digital image processing, for example in medical imaging [1, 2] where it can be used in several processes like: characterization of the anatomical changes in the heart during a cardiac cycle, quantification of the gradual atrophy of the brain by ageing, modeling the evolution of anatomic structures during medical treatment, tissue segmentation through medical atlases, correction of artifacts caused by movement in fetal images, among many other [2-4]. According to the analysed deformations, image registration can be divided into two philosophies: rigid registration or parametric, and elastic or non-rigid. In the literature, the problem of rigid registration has been studied extensively [2], where basically a cost function related to the

* This work was supported by CONACYT through grants No. 168140.

$\star \star$ I. Reducindo was supported by CONACyT doctoral scholarship No. 218513.

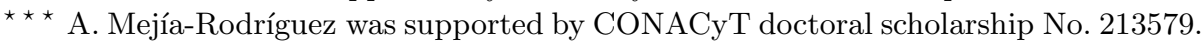

R. Klette, M. Rivera, and S. Satoh (Eds.): PSIVT 2013, LNCS 8333, pp. 36-47, 2014.

(C) Springer-Verlag Berlin Heidelberg 2014 
registration accuracy is minimized that depends on a small set of parameters of a rigid transformation (affine or perspective [5]). The goal of these methods is to obtain the set of parameters describing a geometric transformation between the target and source images that optimizes the similarity metric (e.g. Mutual Information) [3], examples of these algorithms are those based on gradient descent [6], or more recent approaches based on global optimization techniques, such as particle filtering $(\mathrm{PF})$ or genetic algorithms [7 9 ].

On the other hand, the non-rigid registration is, as expected, a more complex and involved problem, especially for multimodal images; however it has a greater number of applications in medical imaging [4]. In the literature, the most common method to solve the elastic registration is by means of splines, where a family of functions are used to approximate the complex non-rigid deformations by seeking their best parameters that optimize a similarity metric; but the main drawback of these methods is their complexity and their high computational cost 10 12]. Meanwhile, some proposals from the literatures are inspired on the optical flow (OF) equations for non-rigid registration [13]. One of these methods is presented in [14], where a non-parametric non-rigid registration modeled as a diffusion process is described. This method, called Demons, alternates between the computation of a force vector related to the variational derivative of the similarity metric, and a regularization process by a simple Gaussian smoothing. Similarly, in [15], it was proposed a non-parametric diffeomorphic registration method based on Demons algorithm. The key idea behind Diffeomorphic Demons (DiffDemons) is to restrict the entire space of deformations vector fields optimized by Demons algorithm to a space of diffeomorphic transformations by combining a recently developed Lie algebra framework on diffeomorphisms, and an optimization procedure for Lie groups. In order to apply these two algorithms (Demons and DiffDemons) for multimodal registration, an histogram matching was performed by normalizing the grayscale values of a source image based on the grayscale values of the reference one at a specified number of quantile values [16]. A most recent proposal to solve the non-rigid registration problem is based on an iterative OF framework in order to find the deformation vector field, after conducting an initial rigid registration using the PF [17]; this method has shown promising results in [18] and [19]. Nonetheless, the problem of this algorithm is its restriction to unimodal images or the necessity of an injective intensity transference function between the target and source images, which is not the case in multimodal medical image registration. In [20], a new proposal is based on mapping the images into a common space, using local variability measures (LVM), where their intensities can be compared, and then to apply an iterative OF in scale space [21], but the problem with this technique is the strong dependence on textures and contrast with respect to the background in the images.

In this context, we propose a new methodology to address the problem of multimodal non-rigid registration under the framework of the ExpectationMaximization (EM) technique [22]. The expectation step is formulated by using an estimation of the joint intensity distribution between the target image and the aligned source image in each iteration of the EM procedure. Next, this distri- 
bution is used to find a new approximation to the deformation vector field whose goal is to improve the registration accuracy between the target and source images. The results in the paper show that the new methodology improved the state of the art algorithms (Demons and DIffDemons) for multimodal non-rigid registration. The paper is organized as follows. In section 2, the non-rigid multimodal image registration based on EM is presented, where the analytical derivations are described in subsections 2.1 and 2.2. Experiments and results are shown in section 3. Finally, in section 4 some conclusions are drawn about this work, as well as directions for future research.

\section{Methodology}

\section{$2.1 \quad$ EM-Approach}

Let $\mathcal{L}$ be a pixel grid where the images to register $I_{1}$ and $I_{2}$ are observed, such that the observation model for each pixel $\mathbf{i}_{k} \in \mathcal{L}$ in the image $I_{1}$ is given by

$$
F\left[I_{1}\left(\mathbf{i}_{\mathbf{k}}\right)\right]=I_{2}\left(\mathbf{i}_{k}+\mathbf{d}\left(\mathbf{i}_{k}\right)\right)+\eta\left(\mathbf{i}_{k}\right) \quad k=1, \ldots, N
$$

where $N=\operatorname{card}(\mathcal{L})$ is the number of pixels in the images, $\mathbf{d}\left(\mathbf{i}_{k}\right)$ is the displacement vector field that aligns the two images $\left(I_{1}, I_{2}\right)$ at each pixel $\mathbf{i}_{k}, \eta\left(\mathbf{i}_{k}\right)$ is Gaussian noise with known probability distribution $P_{\eta}$ (e.g. $\eta\left(\mathbf{i}_{k}\right) \forall \mathbf{i}_{k} \in \mathcal{L}$ are i.i.d. samples of a normal random variable with zero mean and standard deviation $\sigma_{\mathbf{i}_{k}}$ ), and $F[\cdot]$ is a relation which allows to compare the intensity values of the pixels in both images. In fact, one major obstacle in multimodal non-rigid registration is an accurate characterization of $F[\cdot]$. However, as will be described in this section, the EM can overcome this limitation by the assignation of hidden variables [22].

Therefore, to estimate the displacement vector field $\mathbf{d}(\cdot)$ in (11), it is convenient to use a probabilistic approach based on the EM algorithm [22], and establish as hidden variables $Y\left(\mathbf{i}_{k}\right) \triangleq F\left[I_{1}\left(\mathbf{i}_{k}\right)\right] \forall \mathbf{i}_{k} \in \mathcal{L}$, in such a way that we can write the a posteriori probability distribution over the while pixel grid $\mathcal{L}$ as

$$
P\left(\mathbf{d} \mid I_{1}, \tilde{I}_{2}, Y\right)=P\left(\mathbf{d} \mid \tilde{I}_{2}, Y\right),
$$

where $\tilde{I}_{2}\left(\mathbf{i}_{k}\right) \triangleq I_{2}\left(\mathbf{i}_{k}+\mathbf{d}\left(\mathbf{i}_{k}\right)\right)$. Notice that given $Y$, this probability distribution only depends on intensity values of the transformed image $\tilde{I}_{2}$. Now, by using the Bayes' theorem, we can rewrite (2) as

$$
P\left(\mathbf{d} \mid \tilde{I}_{2}, Y\right)=\frac{P\left(\tilde{I}_{2}, Y \mid \mathbf{d}\right) P(\mathbf{d})}{P\left(\tilde{I}_{2}, Y\right)},
$$

but since $P\left(\tilde{I}_{2}, Y\right)$ is a joint intensity distribution that does not depends on $\mathbf{d}$, then it acts a normalizing constant in (3). Thus we can rewrite the previous equation as

$$
P\left(\mathbf{d} \mid \tilde{I}_{2}, Y\right)=\frac{1}{Z} P\left(\tilde{I}_{2}, Y \mid \mathbf{d}\right) P(\mathbf{d})
$$


where $Z \triangleq P\left(\tilde{I}_{2}, Y\right)$. If we assume that each pixel in $\mathcal{L}$ is independent, then the a posteriori probability distribution in (2) can be expressed as

$$
\begin{aligned}
P\left(\mathbf{d} \mid \tilde{I}_{2}, Y\right) & =\prod_{k=1}^{N} P\left(\mathbf{d}\left(\mathbf{i}_{k}\right) \mid \tilde{I}_{2}\left(\mathbf{i}_{k}\right), Y\left(\mathbf{i}_{k}\right)\right) \\
& =\prod_{k=1}^{N} \frac{1}{Z\left(\mathbf{i}_{k}\right)} P\left(I_{2}\left(\mathbf{i}_{k}+\mathbf{d}\left(\mathbf{i}_{k}\right)\right), Y\left(\mathbf{i}_{k}\right) \mid \mathbf{d}\left(\mathbf{i}_{k}\right)\right) P\left(\mathbf{d}\left(\mathbf{i}_{k}\right)\right) .
\end{aligned}
$$

Therefore, by analyzing the a posteriori distribution in (5), the EM framework can be applied by considering the displacement vector field at each pixel $\mathbf{d}\left(\mathbf{i}_{k}\right)$ as the unknown parameters, $Y\left(\mathbf{i}_{k}\right)$ as the hidden variables, and the information of the registered images $\left(I_{1}\left(\mathbf{i}_{k}\right), I_{2}\left(\mathbf{i}_{k}\right)\right)$.

\subsection{The Expectation Step (E)}

Now, the cost function for the expectation step with respect to the hidden variables $Y\left(\mathbf{i}_{k}\right)$ can be written as

$$
\begin{aligned}
Q\left(\mathbf{d}, \mathbf{d}^{(t-1)}\right)=\mathcal{E} & {\left[\log \left\{\prod_{k=1}^{N} \frac{P\left(I_{2}\left(\mathbf{i}_{k}+\mathbf{d}\left(\mathbf{i}_{k}\right)\right), Y\left(\mathbf{i}_{k}\right) \mid \mathbf{d}\left(\mathbf{i}_{k}\right)\right)}{Z\left(\mathbf{i}_{k}\right)} P\left(\mathbf{d}\left(\mathbf{i}_{k}\right)\right)\right\} \mid I_{1}, I_{2}, \mathbf{d}^{(t-1)}\right], } \\
= & \sum_{k=1}^{N} \mathcal{E}\left[\log P\left(I_{2}\left(\mathbf{i}_{k}+\mathbf{d}\left(\mathbf{i}_{k}\right)\right), Y\left(\mathbf{i}_{k}\right) \mid \mathbf{d}\left(\mathbf{i}_{k}\right)\right) \mid I_{1}, I_{2}, \mathbf{d}^{(t-1)}\right] \\
& +\sum_{k=1}^{N} \mathcal{E}\left[\log P\left(\mathbf{d}\left(\mathbf{i}_{k}\right)\right) \mid I_{1}, I_{2}, \mathbf{d}^{(t-1)}\right]-\sum_{k=1}^{N} \mathcal{E}\left[\log Z\left(\mathbf{i}_{k}\right) \mid I_{1}, I_{2}, \mathbf{d}^{(t-1)}\right]
\end{aligned}
$$

where $\mathbf{d}^{(t-1)}$ is an estimate of displacement vector field at a given iteration $(t-1)$ in the EM-algorithm. Moreover, if $Y$ only takes integer values in the set $\mathcal{I}=\{0, \ldots, 255\}$, the cost function $Q\left(\mathbf{d}, \mathbf{d}^{(t-1)}\right)$ can be expressed as

$$
\begin{aligned}
Q\left(\mathbf{d}, \mathbf{d}^{(t-1)}\right)= & \underbrace{\sum_{Y \in \mathcal{I}}\left\{\sum_{k=1}^{N} \log P\left(I_{2}\left(\mathbf{i}_{k}+\mathbf{d}\left(\mathbf{i}_{k}\right)\right), Y\left(\mathbf{i}_{k}\right) \mid \mathbf{d}\left(\mathbf{i}_{k}\right)\right)\right\} P\left(Y \mid I_{1}, I_{2}, \mathbf{d}^{(t-1)}\right)}_{\Gamma\left(\mathbf{d}, \mathbf{d}^{(t-1)}\right)} \\
& +\underbrace{\sum_{k=1}^{N} \log P\left(\mathbf{d}\left(\mathbf{i}_{k}\right)\right)}_{R(\mathbf{d})}+\underbrace{\left(-\sum_{k=1}^{N} \log Z\left(\mathbf{i}_{k}\right)\right)}_{K_{1}} \\
= & \Gamma\left(\mathbf{d}, \mathbf{d}^{(t-1)}\right)+R(\mathbf{d})+K_{1},
\end{aligned}
$$

As a result, the term $\Gamma\left(\mathbf{d}, \mathbf{d}^{(t-1)}\right)$ is commonly called the data term, while $R(\mathbf{d})$ is known as the regularization term. Next, by the observation model in (1), 
conditional intensity distribution related to the hidden variables can be simplified as follows

$$
P\left(Y \mid I_{1}, I_{2}, \mathbf{d}^{(t-1)}\right)=P\left(Y \mid I_{1}, \mathbf{d}^{(t-1)}\right)
$$

Furthermore, if we assume that each element of $Y$ is independent, then the data term can be written as

$$
\begin{aligned}
& \Gamma\left(\mathbf{d}, \mathbf{d}^{(t-1)}\right)=\sum_{Y \in \mathcal{I}}\left\{\sum_{k=1}^{N} \log P\left(I_{2}\left(\mathbf{i}_{k}+\mathbf{d}\left(\mathbf{i}_{k}\right)\right), Y\left(\mathbf{i}_{k}\right) \mid \mathbf{d}\left(\mathbf{i}_{k}\right)\right)\right\} \\
& \times \prod_{m=1}^{N} P\left(Y\left(\mathbf{i}_{m}\right) \mid I_{1}\left(\mathbf{i}_{m}\right), \mathbf{d}\left(\mathbf{i}_{m}\right)^{(t-1)}\right) \\
& =\sum_{Y\left(\mathbf{i}_{1}\right)=0}^{255} \cdots \sum_{Y\left(\mathbf{i}_{N}\right)=0}^{255} \sum_{k=1}^{N}\left\{\log P\left(I_{2}\left(\mathbf{i}_{k}+\mathbf{d}\left(\mathbf{i}_{k}\right)\right), Y\left(\mathbf{i}_{k}\right) \mid \mathbf{d}\left(\mathbf{i}_{k}\right)\right)\right\} \\
& \times \prod_{m=1}^{N} P\left(Y\left(\mathbf{i}_{m}\right) \mid I_{1}\left(\mathbf{i}_{m}\right), \mathbf{d}\left(\mathbf{i}_{m}\right)^{(t-1)}\right) \\
& =\sum_{Y\left(\mathbf{i}_{1}\right)=0}^{255} \cdots \sum_{Y\left(\mathbf{i}_{N}\right)=0}^{255} \sum_{k=1}^{N} \sum_{l=0}^{255} \delta\left(l-Y\left(\mathbf{i}_{k}\right)\right)\left\{\log P\left(I_{2}\left(\mathbf{i}_{k}+\mathbf{d}\left(\mathbf{i}_{k}\right)\right), l \mid \mathbf{d}\left(\mathbf{i}_{k}\right)\right)\right\} \\
& \times \prod_{m=1}^{N} P\left(Y\left(\mathbf{i}_{m}\right) \mid I_{1}\left(\mathbf{i}_{m}\right), \mathbf{d}\left(\mathbf{i}_{m}\right)^{(t-1)}\right) \\
& =\sum_{k=1}^{N} \sum_{l=0}^{255}\left\{\log P\left(I_{2}\left(\mathbf{i}_{k}+\mathbf{d}\left(\mathbf{i}_{k}\right)\right), l \mid \mathbf{d}\left(\mathbf{i}_{k}\right)\right)\right\} \\
& \times \underbrace{\sum_{Y\left(\mathbf{i}_{1}\right)=0}^{255} \cdots \sum_{Y\left(\mathbf{i}_{N}\right)=0}^{255} \delta\left(l-Y\left(\mathbf{i}_{k}\right)\right) \prod_{m=1}^{N} P\left(Y\left(\mathbf{i}_{m}\right) \mid I_{1}\left(\mathbf{i}_{m}\right), \mathbf{d}\left(\mathbf{i}_{m}\right)^{(t-1)}\right)}_{\Theta},
\end{aligned}
$$

where $\mathbf{i}_{m} \in \mathcal{L}$ for all $m=1, \ldots, N$, and $\delta(\cdot)$ represents the Kronecker delta function. In addition, since $P\left(Y\left(\mathbf{i}_{m}\right) \mid I_{1}\left(\mathbf{i}_{m}\right), \mathbf{d}\left(\mathbf{i}_{m}\right)^{(t-1)}\right)$ represents the conditional distribution of $Y\left(\mathbf{i}_{m}\right)$ given $I_{1}\left(\mathbf{i}_{m}\right)$, this distribution can be estimated by using the joint histogram of intensities between the images $I_{1}, \tilde{I}_{2}$ using the displacement vector field $\mathbf{d}^{(t-1)}$. Now, we can simplify the term $\Theta$ by observing that it can be rewritten as

$$
\begin{aligned}
\Theta= & \left(\sum_{Y\left(\mathbf{i}_{1}\right)=0}^{255} \ldots \sum_{Y\left(\mathbf{i}_{N}\right)=0}^{255} \delta\left(l-Y\left(\mathbf{i}_{k}\right)\right) \prod_{m=1, m \neq k}^{N} P\left(Y\left(\mathbf{i}_{m}\right) \mid I_{1}\left(\mathbf{i}_{m}\right), \mathbf{d}\left(\mathbf{i}_{m}\right)^{(t-1)}\right)\right) \\
& \times P\left(l \mid I_{1}\left(\mathbf{i}_{k}\right), \mathbf{d}\left(\mathbf{i}_{k}\right)^{(t-1)}\right) \\
= & \prod_{m=1, m \neq k}^{N}\left(\sum_{Y\left(\mathbf{i}_{m}\right)=0}^{255} P\left(Y\left(\mathbf{i}_{m}\right) \mid I_{1}\left(\mathbf{i}_{m}\right), \mathbf{d}\left(\mathbf{i}_{m}\right)^{(t-1)}\right)\right) P\left(l \mid I_{1}\left(\mathbf{i}_{k}\right), \mathbf{d}\left(\mathbf{i}_{k}\right)^{(t-1)}\right),
\end{aligned}
$$


and since $\sum_{Y\left(\mathbf{i}_{m}\right)=0}^{255} P\left(Y\left(\mathbf{i}_{m}\right) \mid I_{1}\left(\mathbf{i}_{m}\right), \mathbf{d}\left(\mathbf{i}_{m}\right)^{(t-1)}\right)=1$ for any $\mathbf{i}_{m} \in \mathcal{L}$, then the data term in (10) is given now by

$$
\Gamma\left(\mathbf{d}, \mathbf{d}^{(t-1)}\right)=\sum_{k=1}^{N} \sum_{l=0}^{255} \log \left\{P\left(I_{2}\left(\mathbf{i}_{k}+\mathbf{d}\left(\mathbf{i}_{k}\right)\right), l \mid \mathbf{d}\left(\mathbf{i}_{k}\right)\right)\right\} P\left(l \mid I_{1}\left(\mathbf{i}_{k}\right), \mathbf{d}\left(\mathbf{i}_{k}\right)^{(t-1)}\right) .
$$

If we define

$$
P\left(I_{2}\left(\mathbf{i}_{k}+\mathbf{d}\left(\mathbf{i}_{k}\right)\right), l \mid \mathbf{d}\left(\mathbf{i}_{k}\right)\right)=\frac{1}{\sqrt{2 \pi} \sigma_{\mathbf{i}_{k}}} \exp \left\{-\frac{\left(l-I_{2}\left(\mathbf{i}_{k}+\mathbf{d}\left(\mathbf{i}_{k}\right)\right)\right)^{2}}{2 \sigma_{\mathbf{i}_{k}}^{2}}\right\},
$$

where $\sigma_{\mathbf{i}_{k}}$ is the standard deviation of the conditional distribution $P\left(l \mid I_{1}\left(\mathbf{i}_{k}\right)\right.$, $\left.\mathbf{d}\left(\mathbf{i}_{k}\right)^{(t-1)}\right)$, then

$$
\log \left\{P\left(I_{2}\left(\mathbf{i}_{k}+d\left(\mathbf{i}_{k}\right)\right), l \mid \mathbf{d}\left(\mathbf{i}_{k}\right)\right)\right\}=\log \frac{1}{\sqrt{2 \pi} \sigma_{\mathbf{i}_{k}}}-\frac{\left(l-I_{2}\left(\mathbf{i}_{k}+\mathbf{d}\left(\mathbf{i}_{k}\right)\right)\right)^{2}}{2 \sigma_{\mathbf{i}_{k}}^{2}} .
$$

Hence we can rewrite equation (16) as

$$
\Gamma\left(\mathbf{d}, \mathbf{d}^{(t-1)}\right)=K_{2}-\sum_{k=1}^{N} \frac{1}{2 \sigma_{\mathbf{i}_{k}}^{2}} \sum_{l=0}^{255}\left(l-I_{2}\left(\mathbf{i}_{k}+\mathbf{d}\left(\mathbf{i}_{k}\right)\right)\right)^{2} P\left(l \mid I_{1}\left(\mathbf{i}_{k}\right), \mathbf{d}\left(\mathbf{i}_{k}\right)^{(t-1)}\right)
$$

where $K_{2} \triangleq-256 \sum_{k=1}^{N} \log \left(\sqrt{2 \pi} \sigma_{\mathbf{i}_{k}}\right)$. Moreover, this term can be simplified as

$$
\begin{aligned}
\Gamma(\mathbf{d}) & =K_{2}-\sum_{k=1}^{N} \frac{1}{2 \sigma_{\mathbf{i}_{k}}^{2}}\left\{\sigma_{\mathbf{i}_{k}}^{2}+\left(\bar{l}-I_{2}\left(\mathbf{i}_{k}+\mathbf{d}\left(\mathbf{i}_{k}\right)\right)\right)^{2}\right\} \\
& =K_{2}+K_{3}-\sum_{k=1}^{N} \frac{1}{2 \sigma_{\mathbf{i}_{k}}^{2}}\left(\bar{l}-I_{2}\left(\mathbf{i}_{k}+\mathbf{d}\left(\mathbf{i}_{k}\right)\right)\right)^{2} \\
& =K-\sum_{k=1}^{N} \frac{1}{2 \sigma_{\mathbf{i}_{k}}^{2}}\left(\bar{l}-I_{2}\left(\mathbf{i}_{k}+\mathbf{d}\left(\mathbf{i}_{k}\right)\right)\right)^{2},
\end{aligned}
$$

where $K_{3} \triangleq-N / 2$, and $K \triangleq K_{2}+K_{3}$ is a constant value. It is worth noting that the data term $\Gamma$ now depends only on $\mathbf{d}$, since its dependence on $\mathbf{d}^{(t-1)}$ is appended on the average value $\bar{l}$ and variance $\sigma_{\mathbf{i}_{k}}^{2}$, both evaluated with respect the conditional distribution $P\left(l \mid I_{1}\left(\mathbf{i}_{k}\right), \mathbf{d}\left(\mathbf{i}_{k}\right)^{(t-1)}\right)$. Therefore, we can write an equivalent expression for the cost function $Q$ as

$$
Q(\mathbf{d})=K-\sum_{k=1}^{N} \frac{1}{2 \sigma_{\mathbf{i}_{k}}^{2}}\left(\bar{l}-I_{2}\left(\mathbf{i}_{k}+\mathbf{d}\left(\mathbf{i}_{k}\right)\right)\right)^{2}-\lambda \sum_{<k, m>}\left\|\mathbf{d}\left(\mathbf{i}_{k}\right)-\mathbf{d}\left(\mathbf{i}_{m}\right)\right\|_{2}^{2},
$$

that only depends on the vector field $\mathbf{d}$, and its information regarding the previous estimation $\mathbf{d}^{(t-1)}$ is inherited in the first and second order statistics $\bar{l}$ and $\sigma_{\mathbf{i}_{k}}^{2}$. Here, we have used a Gibbs distribution [23] for the regularization term $R(\mathbf{d})=\lambda \sum_{<k, m>}\left\|\mathbf{d}\left(\mathbf{i}_{k}\right)-\mathbf{d}\left(\mathbf{i}_{m}\right)\right\|_{2}^{2}$, where $\lambda>0$ is a parameter which controls the homogeneity of the displacement vector field $\mathbf{d}$, and $\langle k, m\rangle$ stands for the nearest neighbours. 


\subsection{The Maximization Step (M)}

At the maximization step (M), it is possible to estimate the vector field $\mathbf{d}$ at $t$-iteration by an optimization process

$$
\mathbf{d}^{(t) *}=\arg \min _{\mathbf{d}} \bar{Q}(\mathbf{d}),
$$

where

$$
\bar{Q}(\mathbf{d})=\sum_{k=1}^{N} \frac{1}{2 \sigma_{\mathbf{i}_{k}}^{2}}\left(\bar{l}-I_{2}\left(i+\mathbf{d}\left(\mathbf{i}_{k}\right)\right)\right)^{2}+\lambda \sum_{<k, m>}\left\|\mathbf{d}\left(\mathbf{i}_{k}\right)-\mathbf{d}\left(\mathbf{i}_{m}\right)\right\|_{2}^{2} .
$$

It is important to point out that the optimization problem in (22) is non-linear and to derive a feasible solution, we approximate the non-linear terms by using a first order Taylor's expansion [6]

$$
I_{2}\left(\mathbf{i}_{k}+\mathbf{d}\left(\mathbf{i}_{k}\right)\right) \approx I_{2}\left(\mathbf{i}_{k}\right)+\nabla I_{2}\left(\mathbf{i}_{k}\right)^{T} \mathbf{d}\left(\mathbf{i}_{k}\right),
$$

where $\nabla(\cdot)$ and $(\cdot)^{T}$ denote the gradient and transpose operators, respectively. Once expanded the data term, the equation (22) can be approximated by

$$
\bar{Q}(\mathbf{d}) \approx \sum_{k=1}^{N} \frac{1}{2 \sigma_{\mathbf{i}_{k}}^{2}}\left(\bar{l}-I_{2}\left(\mathbf{i}_{k}\right)-\nabla I_{2}\left(\mathbf{i}_{k}\right)^{T} \mathbf{d}\left(\mathbf{i}_{k}\right)\right)^{2}+\lambda \sum_{<k, m>}\left\|\mathbf{d}\left(\mathbf{i}_{k}\right)-\mathbf{d}\left(\mathbf{i}_{m}\right)\right\|_{2}^{2},
$$

where the optimality stationary conditions are derived with respect to the displacements $\mathbf{d}\left(\mathbf{i}_{k}\right)$. Due to the quadratic structure of the cost function $\bar{Q}(\mathbf{d})$, the resulting system of equations is linear and it can be solved by using the Gauss-Seidel algorithm. However, this approximation is accurate only if the displacement vector field $\mathbf{d}\left(\mathbf{i}_{k}\right)$ is small; thus it is suggested to apply first a global parametric multimodal rigid registration; in this work, we use an algorithm based on the Particle Filter (PF) described in [8], in order to obtain a first estimation of the displacement vector field.

In summary, the proposed method to solve the non-rigid multimodal image registration can be described by the next algorithm:

1. Compute a multimodal rigid registration by finding the parameters of an affine transformation through the PF. This affine transformation is used to calculate a first approximation of displacements vector field $\mathbf{d}^{(0)}$.

2. Find the vector field $\mathbf{d}^{(n)}$ in an iterative form departing from the previous estimations $\mathbf{d}^{(0)}+\mathbf{d}^{(1)}+\ldots+\mathbf{d}^{(n-1)}$, by applying the EM-algorithm with the cost function

$$
\begin{aligned}
\bar{Q}\left(\mathbf{d}^{(n)}, \mathbf{d}^{(n-1)}\right) \approx \sum_{k=1}^{N} & \frac{1}{2 \sigma_{\mathbf{i}_{k}}^{2}}\left(\bar{l}-\tilde{I}_{2}^{(n-1)}\left(\mathbf{i}_{k}\right)-\nabla \tilde{I}_{2}^{(n-1)}\left(\mathbf{i}_{k}\right) \mathbf{d}^{(n)}\left(\mathbf{i}_{k}\right)^{T}\right)^{2} \\
& +\lambda \sum_{<k, m>}\left\|\mathbf{d}^{(n)}\left(\mathbf{i}_{k}\right)-\mathbf{d}^{(n)}\left(\mathbf{i}_{m}\right)\right\|_{2}^{2}
\end{aligned}
$$

until a convergence criterion on the magnitude of $\mathbf{d}^{(n)}$ is satisfied; here $\tilde{I}_{2}^{(n-1)}$ $\left(\mathbf{i}_{k}\right) \triangleq I_{2}\left(\mathbf{i}_{k}+\sum_{l=0}^{n-1} \mathbf{d}^{(l)}\left(\mathbf{i}_{k}\right)\right) \forall \mathbf{i}_{k} \in \mathcal{L}$, and $\bar{l}=\mathcal{E}\left\{l \mid I_{1}\left(\mathbf{i}_{k}\right), \sum_{l=0}^{n-1} \mathbf{d}^{(l)}\left(\mathbf{i}_{k}\right)\right\}$. 
3. If the convergence condition is met, then the resulting displacement vector field is given by $\mathbf{d}=\mathbf{d}^{(0)}+\mathbf{d}^{(1)}+\ldots+\mathbf{d}^{(n)}$ and stop, else $n=n+1$ and go to step 2 .

\section{$3 \quad$ Experiments and Results}

The following three experiments show the capacity of the proposed algorithm to register images having different gray intensities. For these examples, the regularization parameter $\lambda$ is fixed to 150. In the proposed approach, the stopping condition in the iterative procedure is defined in terms of the average value of the Euclidean norm of the displacement vector field $\mathbf{d}^{(n)}$; then, the algorithm stops when this value is less than $10^{-3}$.

\subsection{Elastic Image Registration with Intensity Variation}

In the first example, we use two MRI images having not only gray intensity differences, but also different brain structures morphology, as it is shown in pictures 1. (a) (source) and 1.(b) (target). The images resolution is $256 \times 256$. In order to visualize the misalignments between the original images, the source image is shown in green and the target image in red, both in Fig. 1.(c).

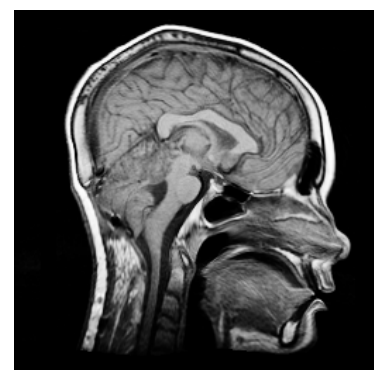

(a)

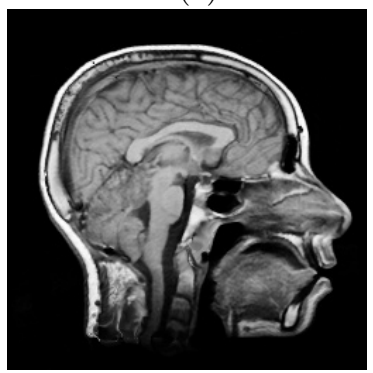

(d)

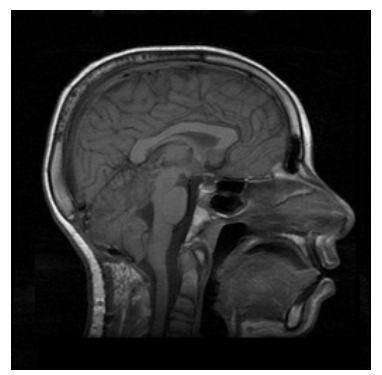

(b)

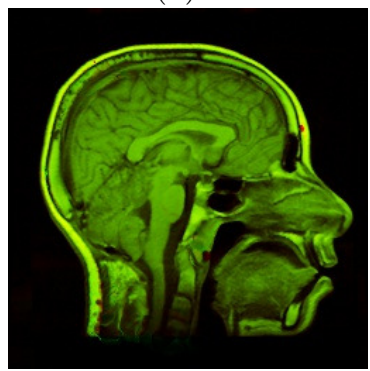

(e)

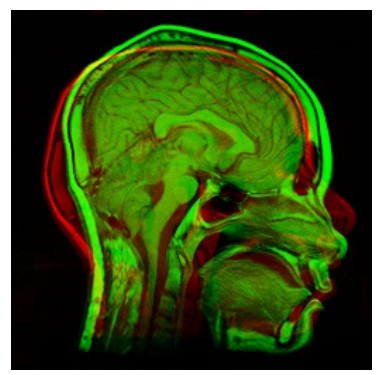

(c)

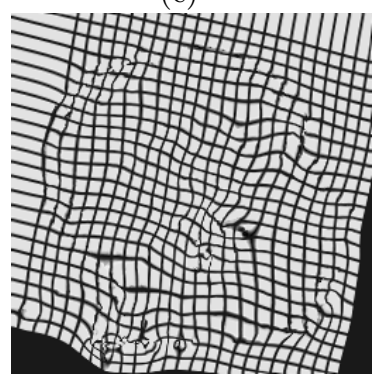

(f)

Fig. 1. Test for Elastic Image Registration with Intensity Variation: (a) Source image, (b) target image, (c) superimposed images, (d) registered source image, (e) superimposed images after registration, and (f) deformation grid field 


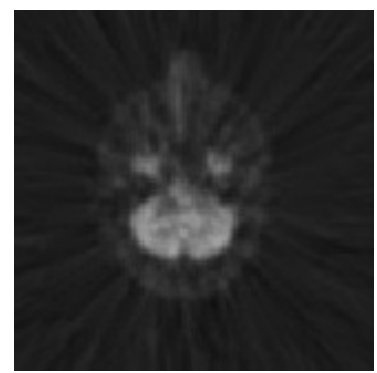

(a)

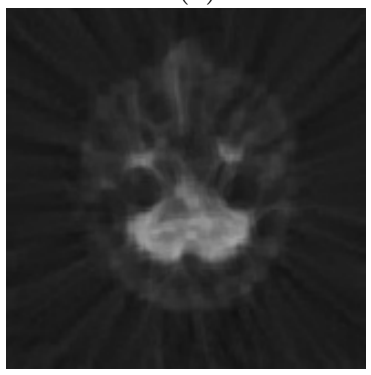

(d)

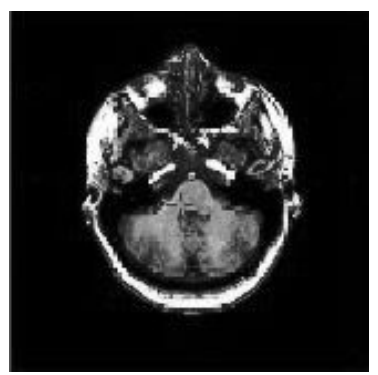

(b)

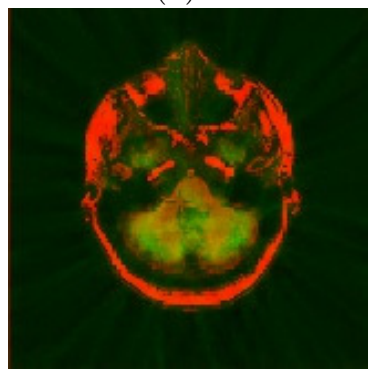

(e)

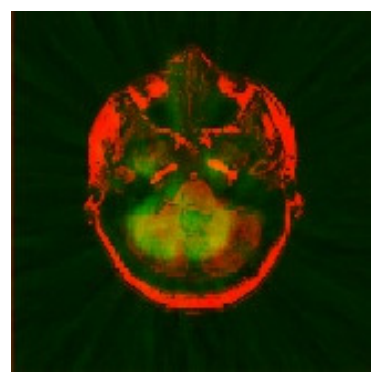

(c)

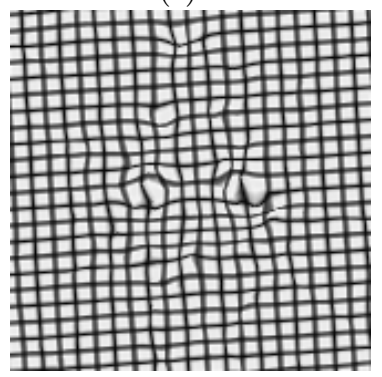

(f)

Fig. 2. Test for Multimodal Image Registration: (a) Source image, (b) target image, (c) superposition of the source and target images, (d) transformed source image after registration, (e) superimposed images after registration, and (f) deformation grid field

The experiment aims to align both MRI images by using our approach. We can observe in Figures 1.(d) and 1. (e) how despite of the differences in structure and gray intensities, the proposed algorithm achieves a very accurate non-rigid multimodal registration.

\subsection{Multi-modality Image Registration}

The second experiment also involves the registration of two images acquired with different techniques: anatomic (MRI) and functional (PET). These images come from very different devices, and by analyzing both images for a specific patient, it is useful to detect functional deficiencies in anatomical structures. The images resolution is $162 \times 162$. The result of the registration process for this experiment is shown in Fig. 2, Note that although the information from the images is very different, qualitatively, the algorithm is able to register them.

\subsection{Registration with Synthetic Deformations}

In order to compare numerically our proposal with recently published algorithms, we performed four experiments where non-rigid image registration algorithms are applied to two MRI images: T1 and T2. In these experiments, we use four 


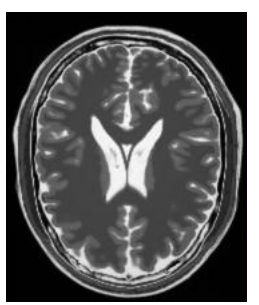

(a)

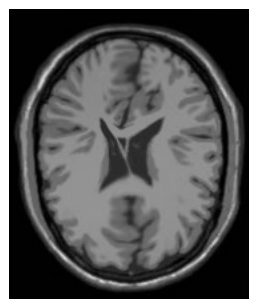

(b)

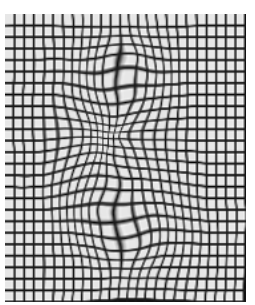

(c)

Fig. 3. Test with Synthetic Deformations: (a) Source T2-image; (b) target T1-image; (c) deformation grid field for GT4

Table 1. Vector field error with respect to the ground truths (Euclidean norm) after non-rigid registration

\begin{tabular}{ccccc}
\hline \hline \multirow{2}{*}{ Method } & \multicolumn{4}{c}{ Vector Field Error } \\
\cline { 2 - 5 } & \multicolumn{4}{c}{ Average $(\mathrm{mm})$} \\
\cline { 2 - 5 } & GT1 & GT2 & GT3 & GT4 \\
\hline \hline LVM & 2.0592 & 1.6432 & 2.0613 & 2.4284 \\
\cline { 2 - 5 } Demons & 3.7906 & 4.8985 & 5.0919 & 6.3559 \\
\cline { 2 - 5 } DiffDemons & 3.8772 & 6.5214 & 4.9879 & 6.9309 \\
\cline { 2 - 5 } EMNRR & $\mathbf{1 . 2 2 1 2}$ & $\mathbf{1 . 3 2 4 6}$ & $\mathbf{1 . 9 0 1 1}$ & $\mathbf{2 . 1 7 2 2}$ \\
\hline \hline EMNRRMS & $\mathbf{0 . 7 1 9 8} \mathbf{0 . 9 1 6 7}$ & $\mathbf{1 . 8 2 3 1 2}$ & $\mathbf{1 . 2 9 9 8}$ \\
\hline \hline
\end{tabular}

synthetic and known vector fields to deform the T1-image, such that each synthetic field represents a ground truth deformation (named GT1, GT2, GT3, and GT4). Fig. 3. (a) illustrates the original T2-weighted source image; Fig. 3. (b) shows one of the four T1-weighted test images, and Fig. 3. (c) shows its deformation grid field (corresponding to GT4). The images resolution is $217 \times 180$. Table 1 presents the numerical results obtained for 4 non-rigid registration algorithms: Local Variability Measures (LVM) [20], Demons [14], DiffDemons [15], and our proposed approach for EM-non-rigid registration (EMNRR). This table shows the mean error of the displacement vector field estimated for each algorithm. In these results we can notice that, among the four approaches, the best performance in terms of the mean error is given by EMNRR.

Furthermore, it is still possible to improve the results obtained with EMNRR by using a multi-scale framework. For this purpose, the target and source images were sub-sampled at two levels (each dividing the image size by two), and the vector field is firstly estimated at the coarsest scale; the solution at this scale is then used as an initial vector field (multiplying each vector by 2) for solving the non-rigid registration problem at each finer scale. The average errors of the estimated vector fields for the multi-scale version (EMNRRMS) are shown in the fifth row of Table 1, illustrating that the multi-scale version of our proposal EMNRRMS still improves its original version EMNRR. 


\section{Conclusions and Future Work}

We propose in this work a new methodology for non-rigid multimodal image registration based on the Expectation-Maximization approach. The mathematical derivations of our proposal were presented in detail in the paper, where the resulting algorithm is based on quadratic optimization and an estimation of the joint conditional intensity distribution of the registering images. The experiments and results showed a remarkable performance to register different kinds of images. We evaluated the algorithm primarily with medical imaging, where it was highlighted as a good alternative for multimodal elastic medical image registration.

For future work, it is pursued to carry out an adaptation strategy of the algorithm parameters in order to increase its performance, for example by modifying spatially the regularization term in order to derive a piecewise smooth vector field, which is more consistent with the behaviour of deformations in medical imaging. Also, a more detailed evaluation of the EMNRR and EMNRRMS algorithms is necessary by using medical index errors. Finally, an implementation for volume registration will be also pursued.

\section{References}

1. Zitová, B., Flusser, J.: Image registration methods: a survey. Image and Vision Computing 21, 977-1000 (2003)

2. Pluim, J.P., Fitzpatrick, J.M.: Image registration. IEEE Transactions on Medical Imaging 22(11), 1341-1343 (2003)

3. Pluim, J.P., Maintz, J.B.A., Viergever, M.A.: Mutual-information-based registration of medical images: a survey. IEEE Transactions on Medical Imaging 22(8), 986-1004 (2003)

4. Rueckert, D., Aljabar, P.: Nonrigid registration of medical images: Theory, methods, and applications. IEEE Signal Processing Magazine 27(4), 113-119 (2010)

5. Gonzalez, R.C., Woods, R.E., Eddins, S.L.: Digital Image Processing Using MATLAB, 2nd edn. Gatesmark Publishing (2009)

6. Nocedal, J., Wright, S.J.: Numerical Optimization, 2nd edn. Springer (2006)

7. Das, A., Bhattacharya, M.: Affine-based registratiom of ct and mr modality images of human brain using multiresolution approaches: comparative study on genetic algorithm and particle swarm optimization. Neural Computing and Applications

8. Arce-Santana, E.R., Campos-Delgado, D.U., Alba, A.: Affine image registration guided by particle filter. IET Image Processing 6(5), 455-462 (2002)

9. Reducindo, I.: Registro rígido de imágenes guiado por filtro de partículas. Master's thesis, Universidad Autónoma de San Luis Potosí (December 2010)

10. Xuan, J., Wang, Y., Freedman, M.T., Adali, T., Shields, P.: Nonrigid medical image registration by finite-element deformable sheet-curve models. International Journal of Biomedical Imaging, 1-9 (2006)

11. Serifović-Trbalić, A., Demirović, D., Prljaca, N., Szekely, G., Cattin, P.C.: Intensity-based elastic registration incorporating and isotropic landmark erros and rotational information. International Journal of Computer Assisted Radiology and Surgery 4(5), 463-468 (2009) 
12. Klein, A., Andersson, J., Ardekani, B.A., Ashburner, J., et al.: Evaluation of 14 nonlinear deformation algorithms applied to human brain mri registration. Neuroimage 46(3), 786-802 (2009)

13. Horn, B.K., Schunck, B.G.: Determining optical flow, Artificial Intelligence 17

14. Thirion, J.P.: Image matching as a diffusion process: an analogy with maxwells demons. Med. Image Anal. 2(3), 243-260 (1998)

15. Vercauteren, T., Pennec, X., Perchant, A., Ayache, N.: Idiffeomorphic demons: efficient non-parametric image registration. Neuroimage 45(1), 561-572 (2009)

16. Laszlo, G.N., Xuan, K.U.: New variants of a method of mri scale standardization. IEEE Tran. on Med. Imaging 19(2), 143-150 (2000)

17. Arce-Santana, E., Campos-Delgado, D.U., Alba, A.: A non-rigid multimodal image registration method based on particle filter and optical flow. In: Bebis, G., et al. (eds.) ISVC 2010, Part I. LNCS, vol. 6453, pp. 35-44. Springer, Heidelberg (2010)

18. Reducindo, I., Arce-Santana, E.R., Campos-Delgado, D.U., Alba, A.: Evaluation of multimodal medical image registration based on particle filter. In: Int. Conf. on Electrical Eng., Computing Science and Automatic Control

19. Mejia-Rodriguez, A., Arce-Santana, E., Scalco, E., Tresoldi, D., Mendez, M., Bianchi, A., Cattaneo, G., Rizzo, G.: Elastic registration based on particle filter in radiotherapy images with brain deformations. In: 2011 Annual International Conference of the IEEE on Engineering in Medicine and Biology Society (EMBC), pp. 8049-8052 (2011), http://dx.doi.org/10.1109/IEMBS.2011.6091985 doi:10.1109/IEMBS.2011.6091985

20. Reducindo, I., Arce-Santana, E.R., Campos-Delgado, D.U., Vigueras-Gómez, F.: Non-rigid multimodal image registration based on local variability measures and optical flow. In: 2012 Annual International Conference of the IEEE on Engineering in Medicine and Biology Society, EMBC (2012)

21. ter Haar Romeny, B., Florack, L., Koenderink, J., Viergever, M.: Scale space: Its natural operators and differential invariants. In: Colchester, A.C.F., Hawkes, D.J. (eds.) IPMI 1991. LNCS, vol. 511, pp. 239-255. Springer, Heidelberg (1991)

22. Dempster, A.P., Laird, N.M., Rubin, D.B.: Maximum likelihood from incomplete data via the em algorithm. Journal of the Royal Statistical Society Series b 39(1), 1-38 (1977)

23. Geman, D., Geman, S.: Stochastic relaxation, gibbs distribution and the bayesian restoration of images. IEEE Trans. Pattern Analysis and Machine Learning Intelligence 6(6), 721-741 (1984) 